\title{
Trajectory Generation for the N-trailer problem using Goursat Normal Form*
}

\author{
D. Tilbury ${ }^{\dagger}$ and R. M. Murray ${ }^{\ddagger}$ and S. Sastry \\ Electronics Research Laboratory \\ Department of Electrical Engineering and Computer Sciences \\ University of California \\ Berkeley, CA 94720
}

In this paper, we show how the machinery of exterior differential systems can be used to help solve nonholonomic motion planning problems. Since the Goursat normal form for exterior differential systems is dual to chained form for vector fields, we solve the problem of steering a mobile robot with $n$ trailers by converting the system into chained form, doing the path-planning in the chained form coordinates, and converting the path back into the original coordinates. Simulations of the $N$-trailer system parallel parking and backing into a loading dock are included.

\section{Introduction}

In this paper, we consider and solve the motion planning problem for a car-like mobile robot pulling $n$ trailers. This system has been an important canonical example for the work on nonholonomic motion planning ever since it was posed in $[10,16]$, because each trailer adds one dimension to the state space of the system (representing its angle with respect to the inertial frame) and one nonholonomic constraint. Regardless of the number of trailers attached, the general system al ways has two degrees of freedom, corresponding to driving and steering for the front car. It has been shown that every point in the state space is reachable, i.e. that the system is completely controllable [10]. Previous work on motion planning for mobile robots can be found in $[1,5,9,12,17,18,21]$ and the references therein. The question that is answered in this paper is one of constructive controllability; explicit open loop controls for steering the car with $n$ trailers from an initial to a final position are given.

In the formulation of exterior differential systems, each constraint that the wheels must roll without slipping is a one-form on the config uration manifold. The collection of these one-forms $I=\left\{\alpha^{1}, \ldots, \alpha^{\alpha}\right\}$ will generate a differential ideal which is referred to as a Pfaffian system. These systems and their properties were first studied by Pfaff in the early 1800's; a large body of work on Pfaff's problem can be found in the literature (see [2] for a historical overview). The formulation of the $\boldsymbol{N}$-trailer problem as a Pfaffian system allows us to draw on classical results on classification and canonical forms, including the Goursat normal form for Pfaffian systems of codimension 2.

After a definition of Pfaffian systems in Section 2, we examine the Pfaffian system associated with a mobile robot towing $n$ trailers. We show in Section 3 that this system can be converted into Goursat's normal form or equivalently chained form. Section 4 is devoted to presenting methods for steering systems in chained form. Three different methods are presented using as inputs sinusoids, piecewise constant inputs (as in [14]) and polynomials. Finally, we apply some of these steering methods to the $N$-trailer example, and display the results in Section 5 .

\section{Exterior Differential Systems}

In this section we give a brief description of some of the tools from exterior differential systems which we will use in the sequel; more detail can be found in $[2,15,22]$. Given a manifold $M$ of dimension $n$, the cotangent space of $M$ at a point $x$ is a vector space of dimension $n$, denoted $T_{x}^{*} M$. The vector space $\Omega^{p}\left(T_{x}^{*} M\right)$ consists of all $p$-forms constructed from cotangent vectors in $T_{x}^{*} M$. By attaching the vector space $\Omega^{p}\left(T_{x}^{*} M\right)$ to each point $x \in M$, we get a bundle structure on $M$, which we write as $\Omega^{p}(M)$. We call a element $\omega \in \Omega^{p}(M)$ an exterior differential $p$-form on $M$.

The research of D. Tilbury and S. Sastry was supported in part by NSF under IRJ-9014490. The work of R. Murray was supported in part by a grant from the Powell Foundation. D. Tilbury would also like to acknowledge an AT\&T Ph.D. Fellowship for partial support of this work.

tdawntoeecs.berkeley.ed

tAddress: Department of Mechanical Engineering, California Institute of Technology, Peapenar CA 91125
Relative to a local coordinate chart, we describe the cotangent bundle by choosing a local basis: $T^{*} M=\operatorname{span}\left\{d x_{1}, \ldots, d x_{n}\right\}$. Let $\Omega(M)$ be the algebra of exterior differential forms on $M$. The exterior derivative on $\Omega(M)$ is the unique map $d: \Omega^{r} \rightarrow \Omega^{r+1}$ which satisfies the following properties:

1. If $f \in \Omega^{0}(M)=C^{\infty}(M)$ then $d f=\sum \frac{\partial l}{\partial x_{i}} d x$

(relative to a local coordinate chart).

2. If $\theta \in \Omega^{r}, \sigma \in \Omega^{*}$ then $d(\theta \wedge \sigma)=d \theta \wedge \sigma+(-1)^{r} \theta \wedge d \sigma$.

3. $d^{2}=0$.

Formally, an exterior differential system is given by an ideal $\mathcal{I} \subset$ $\Omega(M)$ that is closed under exterior differentiation. Recall that an ideal $\mathcal{I}$ satisfies

$$
\alpha \in \mathcal{I}, \beta \in \Omega(M) \quad \Longrightarrow \quad \alpha \wedge \beta \in \mathcal{I} .
$$

We will be primarily interested in the special case of exterior differential systems which are generated by a set of nonholonomic constraints and we focus on that case here.

A Pfaffian system is an exterior differential system which is generated by a set of linearly independent one-forms. Let $I$ be a codistribution spanned by a set of linear independent one-forms $\left\{\alpha^{i}\right\}, i=1, \ldots, s$. The ideal generated by $I$ is

$$
I=\{I\}=\left\{\sigma \in \Omega: \sigma \wedge \alpha^{1} \cdots \wedge \alpha^{s}=0\right\} .
$$

For an ideal generated by a set of one-forms, each element in the ideal has the form $\xi=\sum_{j=1}^{b} a_{i j} \theta^{j} \wedge \alpha^{j}$ for some $\theta^{j} \in \Omega$.

We will also use the notion of congruence. Given two forms $\omega, \xi \in$ $\Omega$, we write $\omega \equiv \xi \bmod \mathcal{I}$ if there exists an exterior form $\eta \in \mathcal{I}$ such that $\omega=\xi+\eta$. If $I$ is a set of one-forms (and hence not an ideal) then we write $\omega \equiv \xi \bmod I$ if there exist exterior forms $\alpha \in I$ and $\eta \in \Omega$ such that $\omega=\xi+\eta \wedge \alpha$. It follows that if $I$ is the generator set for an ideal $\mathcal{I}$, then $\omega \bmod \mathcal{I}=\omega \bmod I$. In the case that $\mathcal{I}$ is generated by one-forms $\left\{\alpha^{i}\right\}$, we will often make use of the relationship

$$
\omega \bmod \mathcal{I} \equiv 0 \Longleftrightarrow \omega=\sum \theta_{i} \wedge \alpha^{i} \quad \text { for some } \theta_{i} \in \Omega \text {. }
$$

Let $I=\operatorname{span}\left\{\omega^{1}, \ldots, \omega^{s}\right\}$ be a smooth codistribution on $M$. The exterior derivative induces a mapping $\delta: I \rightarrow \Omega^{2}(M) / I$ :

$$
\delta: \lambda \mapsto d \lambda \bmod I \in \Omega^{2}(M) \text {. }
$$

The mapping $\delta$ is a linear mapping over $C^{\infty}(M)$. It follows that the kernel of $\delta$ is a codistribution on $M$ (i.e. at each point $p \in M$, the kernel of $\delta$ is a linear subspace of $\left.T_{p}^{*} M\right)$. We call this subspace $I^{(1)}$, the first derived system of $I$ :

$$
I^{(1)}=\operatorname{ker} \delta=\{\lambda \in I: d \lambda \bmod I \equiv 0\} .
$$

We can represent $I^{(1)}$ using a set of one-forms, but it is important to note that the basis for $I^{(1)}$ may not be a simple subset of the basis for $I$.

Since $I^{(1)}$ is itself a smooth codistribution on $M$, we can continue this construction and generate a nested sequence of codistributions

$$
I=I^{(0)} \supset I^{(1)} \supset \cdots \supset I^{(N)} \text {. }
$$

If the dimension of each $I^{(i)}$ is constant, then this construction terminates for some finite integer $N$. In this case, we call equation (1) the derived flag of $I$ and $N$ the derived length.

$I^{(N)}$ is always integrable since by definition $d I^{(N)} \bmod I^{(N)} \equiv 0$. $I^{(N)}$ is the largest integrable subsystem contained in $I$. Thus if $I^{(N)}$ is not empty, then there exist functions $h_{1}, \ldots, h_{r}$ such that $\left\{d h_{i}\right\} \subset$ $\{I\}$. In the context of control theory, this means that the system is 
not controllable since there exist algebraic functions which provide a foliation of the state space and it is impossible to move from one leaf of the foliation to another. The converse of this controllability result is provided by Chow's Theorem, which says that there exists a path between any two points which satisfies the constraints if and only if $I^{(N)}=\{0\}$.

We say that a basis $\left\{\alpha^{i}\right\}$ is adapted to the derived flag if

$$
I^{(i)}=\left\{\alpha^{1}, \ldots, \alpha^{i}\right\} \text {, }
$$

where $s_{i}$ is a strictly decreasing sequence of integers. In other words, an adapted basis is one in which the derived systems are calculated by dropping elements from the end of the basis.

The simplest type of normal form for a nonholonomic system in volves a single constraint.

Theorem 1 (Pfaff's problem) Suppose $\alpha$ is a one-form which satisfies $(d \alpha)^{r+1} \wedge \alpha=0,(d \alpha)^{r} \wedge \alpha \neq 0$. Then there exist coordinates such that

$$
\alpha=d x_{1}+x_{3} d x_{3}+\cdots+x_{2 r} d x_{2 r+1} .
$$

In the $r=1$ case, the proof reduces to proving that there exist two functions $f_{1}$ and $f_{2}$ which satisfy

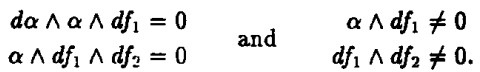

Given $f_{1}$ and $f_{3}, \alpha$ can be scaled such that

$$
\alpha=d f_{2}+g d f_{1}=: d x_{1}+x_{2} d x_{3} .
$$

The Pfaff theorem guarantees that these equations have a solution (it need not be unique).

We now turn to the more general case of $n-2$ constraints on an $n$-dimensional manifold $M$. Let $I$ be a codistribution on $M$ whose derived flag satisfies $\operatorname{dim} I^{(i)}=n-i-2$.

Theorem 2 (Goursat normal form) Let $U$ be an open subset of $\mathbf{R}^{n}$ and $I=\left\{\alpha^{1}, \ldots, \alpha^{2}\right\}$ be a collection of $s=n-2$ smooth, linearly independent one-forms defined on $U$. If there exists a one-form $\pi \neq 0$ mod I such that

$$
\begin{aligned}
& d \alpha^{i} \equiv-\alpha^{i+1} \wedge \pi \bmod \alpha^{1}, \ldots, \alpha^{i} \quad i=1, \ldots, s-1 \\
& d \alpha^{s} \neq 0 \bmod I
\end{aligned}
$$

then there exists a set of coordinates $\xi$ such that

$$
I=\left\{d \xi_{n}-\xi_{n-1} d \xi_{1}, \ldots, d \xi_{3}-\xi_{2} d \xi_{1}\right\} .
$$

A complete proof of this theorem can be found in [2]. It can be summarized in the following algorithm for converting a system into Goursat form (see [7] for the feedback linearization version of this algorithm, on which this is based).

Algorithm 1 Given a codistribution $I=\left\{\omega^{1}, \ldots, \omega^{*}\right\}$ with $s=n-2$, the following steps are required:

1. Construct a basis $I=\left\{\alpha^{1}, \ldots, \alpha^{*}\right\}$ which is adapted to the derived flag. Check the Goursat congruences to ensure they are satisfied for some $\pi$.

2. It follows from the congruences that $\alpha^{1}$ satisfies $\left(d \alpha^{1}\right)^{2} \wedge \alpha^{1}=0$ and hence the proof of Pfaff's theorem can be used to find coordinates such that

$$
\alpha^{2}=d \xi_{n}-\xi_{n-1} d \xi_{1}
$$

3. The remaining coondinates are determined by simple differentiation. Given $\xi_{i}$ we determine $\xi_{i-1}$ by algebraically solving the equation

$$
\alpha^{n-i+1} \equiv d \xi_{i}+\xi_{i-1} d \xi_{1} \quad \bmod \alpha^{1}, \ldots, \alpha^{n-i+1}
$$

The proof of Goursat's theorem is to essentially show that this equation always has a solution.

3 The N-trailer system

In this section, we define the Pfaffian system (set of one-forms which represent the velocity constraints) for the $N$-trailer problem and calculate its derived flag. We then show how the system can be converted into either Goursat normal form (following Theorem 2 and Algorithm 1) or its dual, chained form. Although the calculations in this section assume a particular configuration of the mobile robot and trailer system, we will show that our model is general enough to encompass not only the specific choice we have made but also a front-wheel drive car pulling trailers and the luggage trains found in airports.

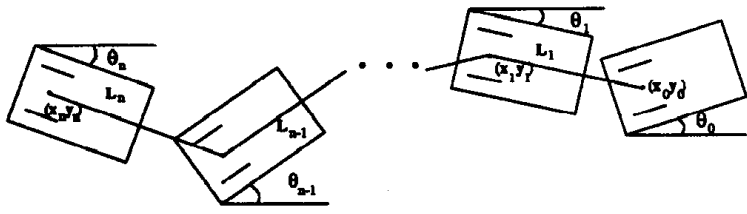

Figure 1: The mobile robot Hilare with $n$ trailers.

\subsection{The rolling constraints and derived flag}

Consider a mobile robot such as Hilare ${ }^{1}$ with $n$ trailers attached, as in Figure 1. Each trailer is attached to the body in front of it by a rigid bar, and each set of wheels is constrained to roll without slipping. The trailers are assumed to be identical, but to have possibly different link lengths $L_{i}$. The $x, y$ coordinates of a midpoint between the two wheels are referred to as $\left(x_{i}, y_{i}\right)$ and the hitch angles (all measured with respect to the horizontal) are $\theta_{i}$. The connections between the bodies give rise to the following constraints:

$$
\begin{aligned}
& x_{i}=x_{i-1}-L_{i} \cos \theta_{i} \\
& y_{i}=y_{i-1}-L_{i} \sin \theta_{i},
\end{aligned}
$$

$i=1,2, \ldots, n$ for the general case with $n$ trailers. These constraints are holonomic and will reduce the dimension of the configuration space, since the positions $\left(x_{i}, y_{i}\right)$ for $i \geq 1$ can be expressed in terms of $x_{0}, y_{0}, \theta_{0}, \ldots, \theta_{i}$. By symmetry, $\left(x_{i}, y_{i}\right)$ for $i<n$ can also be expressed in terms of $x_{n}, y_{n}, \theta_{n}, \theta_{n-1}, \ldots, \theta_{i}$. For our purposes it will be far more useful to use as configuration space variables the $x, y$ coordinates of a point on the $n^{\text {th }}$ trailer and the $n+1$ hitch angles: $x_{n}, y_{n}, \theta_{n}, \ldots, \theta_{0}$ because the calculations that follow are vastly simplified." We will refer to the state space as $x=\left(x_{n}, y_{n}, \theta_{n}, \ldots, \theta_{1}, \theta_{0}\right)$. We have assumed that the bodies are connected between the midpoints of the two sets of rear wheels; it should be noted that if the trailers are hitched behind the rear axle, the equations will not simplify as shown here.

The wheels of the robot and trailers are constrained to roll without slipping; this implies that the velocity of each body in the direction perpendicular to its wheels must be zero. We model each pair of rear wheels as a single wheel at the midpoint of the axle, and state the non-slipping conditions in terms of coordinates, beginning with the $n^{\text {th }}$ trailer:

$$
\alpha^{1}\left(x_{n}, y_{n}, \theta_{n}, \ldots, \theta_{0}\right)=\sin \theta_{n} d x_{n}-\cos \theta_{n} d y_{n} .
$$

To write the other rolling constraints, we define $v_{i}$ to be the magnitude of the velocity of the $i^{\text {th }}$ trailer. The direction of motion of the $(i+1)^{t}$ trailer and consequently the direction of $v_{i+1}$, if its wheels are rolling without slipping, is along the direction of the hitch joining the $(i+1)^{\text {at }}$ body to the $i^{t h}$ body. Since the bodies are linked together by rigid rods, it follows that the projection of $v_{i}$ onto the line of the hitch is equal to $v_{i+1}$. Thus, we have that

$$
v_{i+1}(x)=\cos \left(\theta_{i+1}-\theta_{i}\right) v_{i}(x) \text {. }
$$

Also, we have that the velocity of the $n^{\text {th }}$ trailer $v_{n}$ is given by

$$
v_{n}(x)=\cos \theta_{n} \dot{x}_{n}+\sin \theta_{n} \dot{y}_{n} \text {. }
$$

In the sequel we will need to use $v_{n}$ as a one form (i.e. we will need to use $v_{n} d t$ ) and we denote this by abuse of notation as:

$$
v_{n}(x)=\cos \theta_{n} d x_{n}+\sin \theta_{n} d y_{n} .
$$

We may now recursively write down the rolling without slipping constraints for all the trailers. The velocity of each trailer has a component due to the velocity $v_{i+1}$ of the previous trailer and a component $L_{i+1} \dot{\theta}_{i+1}$ due to the rotation of the hitch. The relative geometry of this situation is illustrated in Figure 2. The component of $v_{i+1}$ in the direction perpendicular to the wheel base is $v_{i+1} \sin \left(\theta_{i}-\theta_{i+1}\right)$ and the component of $L_{i+1} \dot{\theta}_{i+1}$ in this perpendicular direction is $L_{i+1} \dot{\theta}_{i+1} \cos \left(\theta_{i}-\theta_{i+1}\right)$. If the $i^{\text {th }}$ trailer rolls without slipping then we must have

$$
0=L_{i+1} \dot{\theta}_{i+1} \cos \left(\theta_{i+1}-\theta_{i}\right)-v_{i+1} \sin \left(\theta_{i+1}-\theta_{i}\right) \text {. }
$$

\footnotetext{
${ }^{1}$ The Bilare family of mobile robots resides at LAAS in Toulouse, see for example $[4,8]$.

${ }^{2}$ The intuition for this comes from the oft repeated dictum: "when backing up a car with a trailer, keep your eye on the hind part of the trailer". Of course, the generalization to this dictum is: when driving a car with $n$ trailers keep your eye on the endpoint of the $n^{\text {th }}$ trailer.
} 


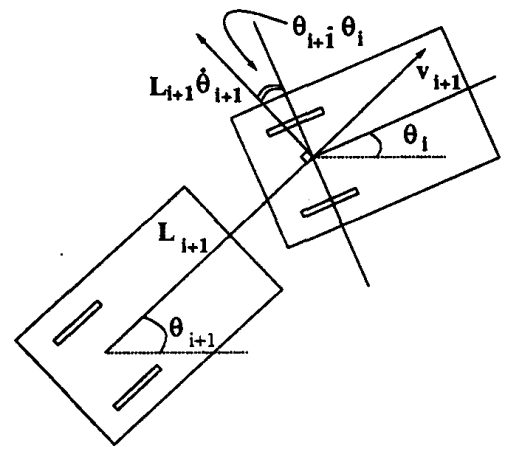

Figure 2: Showing the angles and velocities of the $i^{\text {th }}$ trailer

Dividing through the equation (9) by $\cos \left(\theta_{i+1}-\theta_{i}\right)$ yields the form constraint for $n-1 \geq i \geq 0$ :

$$
\alpha^{n+1-i}(x)=L_{i+1} d \theta_{i+1}-\tan \left(\theta_{i+1}-\theta_{i}\right) v_{i+1}=0 .
$$

Note that we have used the one form version of $v_{i+1}$ in equation (10).

The forms $\alpha^{1}(x), \alpha^{2}(x), \ldots, \alpha^{n+1}(x)$ represent the constraints that the wheels of the $n^{t h},(n-1)^{n t}, \ldots, 0^{t h}$ trailer (i.e. the cab), respectively roll without slipping. They are given by the formulas (10) with the recursion relations (6). Thus, the Pfaffian system for the $N$-trailer problem is:

$$
I=\operatorname{span}\left\{\alpha^{1}, \alpha^{2}, \ldots, \alpha^{n+1}\right\} .
$$

The following theorem gives the derived flag associated with this Pfaffian system.

Theorem 3 (Derived Flag for the $N$-trailer Pfaffian system) Consider the Pfaffian system of the N-trailer system (11) with the one forms $\alpha^{i}$ defined by equations (5) and (10). The one-forms $\alpha^{i}$ are adapted to the derived flag in the following sense:

$$
\begin{aligned}
I^{(0)} & =\operatorname{span}\left\{\alpha^{1}, \alpha^{2}, \ldots, \alpha^{n}, \alpha^{n+1}\right\} \\
I^{(1)} & =\operatorname{span}\left\{\alpha^{1}, \alpha^{2}, \ldots, \alpha^{n}\right\} \\
& \vdots \\
I^{(n)} & =\operatorname{span}\left\{\alpha^{1}\right\} \\
I^{(n+1)} & =\{0\}
\end{aligned}
$$

The proof is by recursion starting from the bottom of the flag of (12), and can be found in [22]. We note that the $I^{(n+1)}=\{0\}$ implies that the $N$-trailer system is completely controllable (by Chow's theorem).

\subsection{Conversion to Goursat Normal Form}

In the preceding subsection, we have shown that the ideal generated by $\alpha^{1}, \ldots, \alpha^{n+1}$ defined in equations (5) and (10) is adapted to the derived flag in the sense of (12). It remains to check whether the $\alpha^{i}$ satisfy the Goursat congruences and if they do, to find a transformation that puts them into the Goursat canonical form.

Theorem 4 (Goursat Congruences for the $N$-trailer system) Consider the Pfaffian system associated with the N-trailer system (11) with the one-forms $\alpha^{i}$ defined by equations (5) and (10). There exists a change of basis of the one forms $\alpha^{i}$ to $\bar{\alpha}^{i}$ which preserves the adapted structure, and a one-form $\pi$ which satisfies the Goursat congruences for this new basis:

$$
\begin{aligned}
d \bar{\alpha}^{i} & \equiv-\bar{\alpha}^{i+1} \wedge \pi \bmod \bar{\alpha}^{1}, \ldots, \bar{\alpha}^{i} \quad i=1, \ldots, n \\
d \bar{\alpha}^{n+1} & \neq 0 \bmod I .
\end{aligned}
$$

The one-form which satisfies these congruences is given by

$$
\pi=\cos \theta_{n} d x_{n}+\sin \theta_{n} d y_{n},
$$

and is equivalent to $v_{n}$, the velocity form of the $n^{\text {th }}$ trailer.

The outline for the proof is first to determine a suitable one-form $\pi$ from the first Goursat congruence, $d \alpha^{1} \equiv-\alpha^{2} \wedge \pi$. Then we construct the new basis elements $\bar{\alpha}^{i}$ one at a time such that they satisfy the rest of the congruences. For this example, we find that these new basis elements are multiples of the original basis elements, and since the original basis is adapted to the derived flag, the new basis is also adapted. For details, we refer the reader to [22].

Now we can follow the steps of the algorithm of Section 2 to find the coordinate transformation that will result in Goursat normal form. Following Algorithm 1, in step 2 we look for possibly non-unique functions $f_{1}, f_{2}$ which satisfy $(2)$, namely

$$
\begin{aligned}
& d \alpha^{1} \wedge \alpha^{1} \wedge d f_{1}=0 \quad \text { and } \quad \alpha^{1} \wedge d f_{1} \neq 0 \\
& \alpha^{1} \wedge d f_{1} \wedge d f_{2}=0 \quad \text { and } \quad d f_{1} \wedge d f_{2} \neq 0
\end{aligned}
$$

Since $\alpha^{1}=\sin \theta_{n} d x_{n}-\cos \theta_{n} d y_{n}$ and $d \alpha^{1}=-\cos \theta_{n} d x_{n} \wedge d \theta_{n}-\sin \theta_{n} \wedge$ $d \theta_{n}$, it follows that $d \alpha^{1} \wedge \alpha^{1}=d x_{n} \wedge d y_{n} \wedge d \theta_{n}$. Thus $f_{1}$ may be chosen to be any function of $x_{n}, y_{n}, \theta_{n}$ exclusively. We now proceed to explain two different solutions of the equations (2):

Transformation 1: Coordinates of the $N^{\text {th }}$ trailer In a choice motivated by Sørdalen [20] we choose $f_{1}=x_{n}$. Then, the second equation of (2) becomes

$$
\sin \theta_{n} d x_{n} \wedge d y_{n} \wedge d f_{2}=0
$$

with the proviso that $d f_{1} \wedge d f_{2} \neq 0$. A non-unique choice of $f_{2}$ is $f_{2}=y_{n}$. For the change of coordinates, we choose

$$
\begin{aligned}
z_{1} & =f_{1}(x)=x_{n} \\
z_{n+3} & =f_{2}(x)=y_{n} .
\end{aligned}
$$

The one form $\alpha^{1}=0$ may be written by dividing through by $\sin \theta_{n}$ as

$$
\alpha^{1}=d y_{n}+\tan \theta_{n} d x_{n}=d z_{n+3}-z_{n+2} d z_{1},
$$

so that $z_{n+2}=-\tan \theta_{n}$. The remaining coordinates are found by solving the equations

$$
\alpha^{i}=d z_{n-i+4}-z_{n-i+3} d z_{1} \bmod \alpha^{1}, \ldots, \alpha^{i-1}
$$

for $i \geq 2$. The details are not particularly insightful and are omitted here.

Transformation 2: Coordinates of the origin seen from the last trailer Yet another choice for $f_{1}$ corresponds to writing the coordinates of the origin as seen from the last trailer. This is reminiscent of a transformation used by Samson [19] in a different context, and is given by

$$
z_{1}:=f_{1}(x)=x_{n} \cos \theta_{n}+y_{n} \sin \theta_{n} .
$$

This has the physical interpretation of being the origin of the reference frame when viewed from a coordinate frame attached to the $n^{\text {th }}$ trailer. It satisfies the first of the equations of (2) simply by virtue of the fact that it is a function of $x_{n}, y_{n}, \theta_{n}$. It may be verified that a choice of $f_{2}$ (non-unique - we got it by guess work!) given by

$$
z_{n+3}:=f_{2}=x_{n} \sin \theta_{n}-y_{n} \cos \theta_{n}-\theta_{n} z_{1}
$$

satisfies

$$
\alpha^{1} \wedge d f_{1} \wedge d f_{2}=0 .
$$

The remaining coordinates $z_{2}, \ldots, z_{n+2}$ corresponding to this transformation may be obtained from the same procedure as in the previous solution. The details are tedious. ${ }^{3}$ In the next subsection, we discuss yet another technique for obtaining the coordinates for the Goursat normal form.

\subsection{Conversion to Chained Form}

A codimension 2 Pfaffian system, $I=\left\{\alpha^{1}(x), \ldots, \alpha^{n-2}(x)\right\}$, is dual to a two-input drift-free control system:

$$
\Sigma: \quad \dot{x}=g_{1}(x) u_{1}+g_{2}(x) u_{2},
$$

where the vector fields $g_{j}(x)$ span a 2-dimensional distribution $\Delta$ which is annihilated by the one-forms $\alpha^{i}, \alpha^{i}(x) \cdot g_{j}(x)=0$. When we transform an exterior differential system into Goursat normal form, we perform a coordinate transformation $z=f(x)$ and reorder the basis. There is no input per se to a formal exterior differential system, although we can speak of the two degrees of freedom of the system, given by the distribution $\Delta=I^{\perp}$

Chained form is dual to the Goursat normal form presented above. That is, a system with constraints in Goursat normal form can always be written as a control system in chained form by choosing

$$
g_{1}=\frac{\partial}{\partial z_{1}}+z_{2} \frac{\partial}{\partial z_{3}}+\cdots+z_{n-1} \frac{\partial}{\partial z_{n}} \quad g_{2}=\frac{\partial}{\partial z_{2}}
$$

${ }^{3}$ Readers interested in the details of the transformation may obtain it from the first author by email or regular mail. 
as a basis for the distribution annihilated by $I$. Thus, we can formulate the problem of finding a basis for the constraints which is in Goursat form as the problem of finding a feedback transformation to convert a system to chained form. In the previous section, we described a method for converting the $N$-trailer exterior differential system into Goursat normal form. We now show how a similar procedure can be used to transform the control system corresponding to the $N$-trailer system into chained canonical form.

Proposition 5 Consider an $N$-trailer system with $n+1$ rolling constrains $\alpha^{i}$ given by (5) and (10). A basis for the distribution $\Delta$ which is annihilated by these one-forms $\left\{\alpha^{d}, \ldots, \alpha^{n+1}\right\}$ is given by

$$
g_{1}=\left[\begin{array}{c}
\cos \theta_{n} \\
\sin \theta_{n} \\
\frac{1}{L_{n}} \tan \left(\theta_{n-1}-\theta_{n}\right) \\
\vdots \\
\frac{1}{L_{1}} \prod_{i=2}^{n} \sec \left(\theta_{i-1}-\theta_{i}\right) \tan \left(\theta_{0}-\theta_{1}\right) \\
0
\end{array}\right] \quad g_{2}=\left[\begin{array}{c}
0 \\
0 \\
0 \\
\vdots \\
0 \\
1
\end{array}\right] .
$$

For the proof, we refer the reader to [22]. Although there are many different choices of $g_{1}, g_{2}$ which will span $\Delta$, the ones which we have chosen are natural in the sense that when the nonholonomic control system is written as:

$$
\dot{x}=g_{1}(x) u_{1}+g_{2}(x) u_{2}
$$

the input functions have the physical meanings: $u_{1}=v_{n}$ is the linear velocity of the $n^{\text {th }}$ trailer, and $u_{2}=\omega$ is the rotational velocity of the lead car. From a practical point of view, we have control only on the velocity $v_{0}$ of the lead car given in terms of $v_{n}$ by

$$
v_{0}=\sec \left(\theta_{0}-\theta_{1}\right) \sec \left(\theta_{1}-\theta_{2}\right) \cdots \sec \left(\theta_{n-1}-\theta_{n}\right) v_{n} .
$$

This is merely an input transformation, and will not change any of the properties of the chained form system.

We will now derive the coordinate transformations and changes of input required to put the system into chained form. Recall that a system in chained canonical form is defined to be

$$
\begin{aligned}
\dot{z}_{1} & =u_{1} \\
\dot{z}_{2} & =u_{2} \\
\dot{z}_{3} & =z_{2} u_{1} \\
& \vdots \\
\dot{z}_{m} & =z_{m-1} u_{1} .
\end{aligned}
$$

We note that the functions $z_{1}(t)$ and $z_{m}(t)$ will completely define all the state variables of a chained-form system, ${ }^{4}$ since the other $m-2$ states and the two inputs can be determined from the equations:

$$
\begin{aligned}
u_{1} & =\dot{z}_{1} \\
z_{i} & =\dot{z}_{i+1} / u_{1} \quad i=m-1, \ldots, 2 \\
u_{2} & =\dot{z}_{2} .
\end{aligned}
$$

Consequently, a coordinate transformation into chained form is completely defined by the first and last coordinates of the chain, $z_{1}$ and $z_{m}$, as functions of the original coordinates $x$ along with equation (15). (The fact that such a transform exists follows from our having verified the Goursat congruences for the $\alpha^{i}$ in the previous subsection.) In general, there are many possible transformations into chained form; two are presented here. These two are exactly the same as those discussed in the previous subsection in the context of the Goursat normal form.

Transformation 1 Originally proposed by Sørdalen [20], and also used in the previous section, is as follows:

$$
\begin{aligned}
z_{1} & =x_{n} \\
z_{n+3} & =y_{n} .
\end{aligned}
$$

The corresponding input transformation is:

$\bar{u}_{1}=\dot{z}_{1}=\cos \theta_{n} v_{n}=\cos \left(\theta_{0}-\theta_{1}\right) \cos \left(\theta_{1}-\theta_{2}\right) \cdots \cos \left(\theta_{n-1}-\theta_{n}\right) \cos \theta_{n} v_{0}$.

'As this paper was being finished it was pointed out to the authors that this situation is referred to by Fliess et al. as flat outputs [6].
The other input $\bar{u}_{2}=\dot{z}_{2}$ is a quite complicated function of $x, v_{0}, \omega$ for the general case with $n$ trailers. However, it is easily verified that

$$
\frac{\partial \bar{u}_{2}}{\partial \omega} \neq 0
$$

implying that the input transformation $\bar{u}=b(x) u$ is nonsingular. The remaining coordinates $z=f(x)$ are defined using equation (15).

It can be checked that this coordinate transformation is valid by looking at the Jacobian,

$$
\left[\frac{\partial z}{\partial x}\right]=\left[\begin{array}{cc|ccc}
1 & 0 & 0 & \cdots & 0 \\
0 & 1 & 0 & \cdots & 0 \\
\hline 0 & 0 & * & & 0 \\
\vdots & \vdots & & \ddots & \\
0 & 0 & * & & *
\end{array}\right],
$$

where the coordinates are written in the order: $x=$ $\left(x_{n}, y_{n}, \theta_{n}, \theta_{n-1}, \ldots, \theta_{0}\right), z=\left(z_{1}, z_{n+3}, z_{n+2}, \ldots, z_{2}\right)$ and $*$ represents some nonzero function. The ordering of the $z$ coordinates was chosen to put the Jacobian matrix in a lower-triangular form, thereby highlighting its nonsingularity. That the Jacobian is nonsingular implies that the map $f: x \rightarrow z$ is a local diffeomorphism.

It should be noted that this coordinate transformation is only defined locally. Since its definition requires a division by $u_{1}$, if any of the factors in $u_{1}$ are zero, the transformation is undefined for that particular configuration. For example, if $\theta_{n}=\pi / 2$, corresponding to the last trailer being at right-angles with the coordinate frame, this coordinate transformation is no longer valid. In addition, if the $i^{\text {th }}$ trailer is jack-knifed, that is to say, for some $1 \leq i \leq n, \theta_{i}=\theta_{i-1} \pm \pi / 2$, the coordinate transformation is also singular.

Transformation 2 Another coordinate transformation which also has some singularities but will allow the trailer to be at any orientation with respect to the coordinate frame, was also detailed in the previous section; we define it here as:

$$
\begin{aligned}
z_{1} & =x_{n} \cos \theta_{n}+y_{n} \sin \theta_{n} \\
z_{n+3} & =x_{n} \sin \theta_{n}-y_{n} \cos \theta_{n}-\theta_{n} z_{1} .
\end{aligned}
$$

The input transformation and the rest of the coordinates follow from (15). Once again, it can be verified that the input transformation has the form:

$$
\left(\begin{array}{l}
\bar{u}_{1} \\
\bar{u}_{2}
\end{array}\right)=\left[\begin{array}{cc}
b_{1,1}(x) & 0 \\
b_{2,1}(x) & b_{2,2}(x)
\end{array}\right]\left(\begin{array}{c}
v_{0} \\
\omega
\end{array}\right)
$$

with $b_{1,1}$ and $b_{2,2}$ nonzero functions of $x$. This implies that the input transformation is nonsingular.

We can show that this coordinate transformation is nonsingular by looking at its Jacobian:

$$
\left[\frac{\partial z}{\partial x}\right]=\left[\begin{array}{ccc|ccc}
\cos \theta_{n} & \sin \theta_{n} & * & 0 & & 0 \\
\sin \theta_{n} & -\cos \theta_{n} & * & & \ddots & \\
0 & 0 & -1 & 0 & & 0 \\
\hline 0 & 0 & * & * & & 0 \\
\vdots & \vdots & \vdots & & \ddots & \\
0 & 0 & * & * & & *
\end{array}\right]
$$

where the coordinates are written in the order: $x=$ $\left(x_{n}, y_{n}, \theta_{n}, \theta_{n-1}, \ldots, \theta_{0}\right)$ and $z=\left(z_{1}, z_{n+3}, z_{n+2}, \ldots, z_{2}\right)$ and $*$ represents any nonzero function. Again, since the Jacobian is nonsingular, the map $f: x \rightarrow z$ is a local diffeomorphism. The singularities in this transformation also occur when division by $u_{1}$ is undefined. This happens when the expression $L_{n}+\left(y \cos \theta_{n}-x \sin \theta_{n}\right) \tan \left(\theta_{n}-\theta_{n-1}\right)=0$, and also when any of the trailers is jack-knifed.

\subsection{Generalizations}

Thus far, we have concentrated our attention on the example of the Hilare mobile robot pulling a chain of trailers. However, here we demonstrate that this model is equivalent (under a coordinate transformation and state feedback) not only to the more familiar system of a frontwheel drive car pulling trailers, but also to the luggage trains commonly found in airports.

The model of the front-wheel drive car is shown in Figure 3. In comparison with the Hilare model, we have added another axle to the front body of the chain, and a variable $\phi$ representing the angle of the front wheels with respect to the car. The length of the wheelbase of the lead car is defined to be $L_{0}$. The luggage cart train is also pulled by a front-wheel drive car. Each of its trailers has two sets of wheels: 


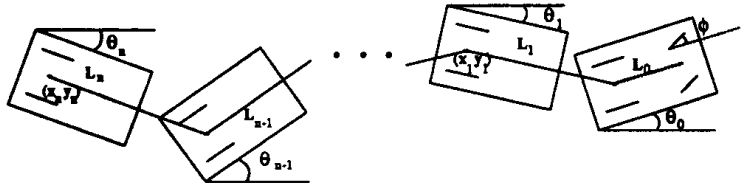

Figure 3: The front-wheel drive car with $n$ trailers.

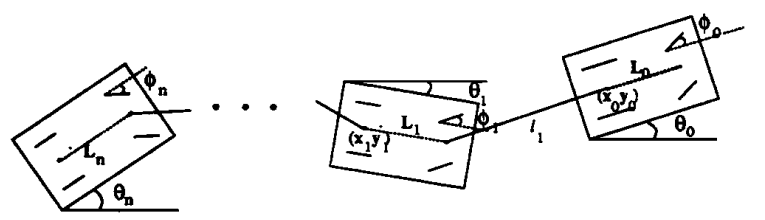

Figure 4: A car pulling $n$ luggage carts.

the front axle can spin freely about its center but the back axle is constrained to be aligned with the trailer (see Figure 4).

The equivalence between the models is most easily seen by looking at the form constraints. Each constraint corresponds to one axle rolling without slipping. Hilare with $n$ trailers has $n+1$ axles; the car with $n$ trailers has $n+2$ axles, and its Pfaffian system is therefore equivalent to that of Hilare pulling $n+1$ trailers. A car pulling $n$ luggage carts has $2 n+2$ axles, and its Pfaffian system is equivalent to that of Hilare pulling $2 n+1$ trailers.

Of course, the states and inputs that we define for these systems are slightly different. By convention, the angle of the front axle is defined relative to the car instead of relative to the coordinate frame. This angle $\phi$ is merely $\theta_{0}-\theta_{1}$ on the Hilare system. The velocity input is the same, assumed to be the linear velocity of the first body (we can define it at either the front or rear axle depending on whether our car is front-wheel drive or rear-wheel drive), but the rotational input is usually taken as $\omega^{\prime}=\dot{\phi}$ the steering wheel velocity. Since in the Hilare case, we can control the velocity of the first body $\omega=\dot{\theta}_{0}$, state feedback can be used to reconcile these differences. There are many choices of vector fields orthogonal to a given Pfaffian system with each choice having a different physical meaning.

\section{Steering Chained Form Systems}

Now that we have seen how to transform an $N$-trailer system into chained form, we examine various methods for steering chained form systems. We assume an $m$-state system, and note that Hilare with $n$ trailers has $n+3$ states, a car with $n$ trailers has $n+4$ states, and a car with $n$ luggage trailers has $2 n+4$ states.

The problem that we address in this section is: Given a system in chained form with an initial state $z^{i}$ and a goal state $z^{f}$, find some control inputs $u_{1}(t), u_{2}(t)$ which will steer the system from $z^{i}$ to $z^{f}$ after some time $T$. The application of these results to the problem of steering the mobile robot with multiple trailers is covered in the next section. We present three methods to steer the chained form system.

\subsection{Sinusoidal inputs}

The first steering method that we consider uses sinusoidal inputs. Steering chained form systems with sinusoids was originally proposed by us in [17]. The method that we have developed here is different from the original algorithm in that it steers all the states in one step, instead of one state at a time.

Given an $m$-state chained form system, it is easily seen that the first two states, $z_{1}$ and $z_{2}$, can be steered from their initial to their final positions using constant inputs over any time period $T$. Of course, the states $z_{3}, \ldots, z_{m}$ will drift as a consequence of this.

By direct integration, it may be verified that a combination of out of phase sinusoids applied to the inputs,

$$
u_{1}(t)=\alpha \sin \omega t \quad u_{2}(t)=\beta \cos k \omega t
$$

applied over one period $T=2 \pi / \omega$, will result in a net motion,

$$
\begin{aligned}
z_{i}(T) & =z_{i}(0) \quad i=1, \ldots, k+1 \\
z_{k+2}(T) & =z_{k+2}(0)+\frac{\alpha^{k} \beta}{k !(2 \omega)^{k}} .
\end{aligned}
$$

The steering algorithm in [17] is step-by-step: It first steers $z_{1}, z_{2}$ to their final position using constant inputs, disregarding the other states.
Then it steers $z_{3}$ to its desired final position using sinusoids, $z_{1}, z_{2}$ will return to their final values. Now $z_{4}$ can be steered, and similarly on down the chain, until all states are at their final positions. This is a simple algorithm that is easy to implement, but can be time-consuming when there are many states to be steered.

We propose instead an "all-at-once" sinusoids method, combining all the frequencies on $u_{2}$ together in one step,

$$
\begin{aligned}
& u_{1}=a_{0}+a_{1} \sin \omega t \\
& u_{2}=b_{0}+b_{1} \cos \omega t+b_{2} \cos 2 \omega t+\cdots+b_{m-2} \cos (m-2) \omega t \text {. (16) }
\end{aligned}
$$

It is no longer as simple to choose appropriate values for the parameters $\left(a_{0}, a_{1}, b_{0}, \ldots, b_{m-2}\right)$ because of the drift that we were able to ignore when we considered each state individually. However, it is still possible to integrate the chained form equations sequentially, finding $z_{1}(t), z_{2}(t), z_{3}(t), \ldots, z_{m}(t)$ which result from the inputs (16) above. The state $z(t)$ is a function of the initial condition $z^{i}$ as well as the input parameters $a_{0}, a_{1}, b_{0}, \ldots, b_{m-2}$. If we evaluate $z(T)$, with $T=2 \pi / \omega$, all the sinusoidal functions will evaluate to either 0 or 1 . By setting $z(T)=z^{f}$ we get a set of $m$ polynomial equations in the $(m+1)$ input parameters $\left(a_{0}, a_{1}, b_{0}, \ldots, b_{m-2}\right)$. The existence of solutions to these equations is guaranteed at least locally around $z^{i}[22]$.

We have dealt with the overparameterization of the input $(m+1$ parameters: $a_{0}, a_{1}, b_{0}, \ldots, b_{m-2}$ and $m$ states) by initially choosing a value for $a_{1}$ and then solving the $m$ equations for the remaining $m$ input parameters. By choosing a fixed value for $a_{1}$, we are requiring $u_{1}$ to go through one period. Since $u_{1}$ roughly corresponds to the driving input in a mobile robot system, paths planned using the sinusoidal method generally have one back-up or speed reversal, corresponding to the zero-crossing of $u_{1}$. Parallel-parking type maneuvers seem particularly well-suited to sinusoidal steering methods.

\subsection{Piecewise Constant Inputs}

The second method we investigate for steering chained form systems uses piecewise constant inputs. This method was originally proposed by Monaco and Normand-Cyrot [14], and was inspired by multirate digital control. It is most easily understood in the context of nonholonomic motion planning simply as piecewise constant inputs.

Consider holding the inputs $u_{1}$ and $u_{2}$ constant over some small time period $[0, \bar{\delta})$,

$$
\begin{aligned}
& u_{1}(\tau)=u_{1,1} \\
& u_{2}(\tau)=u_{2,1} .
\end{aligned} \quad \tau \in[0, \bar{\delta})
$$

The chained form state equations can then be integrated, and evaluated at time $\bar{\delta}$ to yield $z(\bar{\delta})=f\left(z(0), u_{1,1}, u_{2,1}\right)$.

We can now consider another pair of constant inputs on the time interval $[\bar{\delta}, 2 \bar{\delta})$,

$$
\begin{array}{ll}
u_{1}(\tau)=u_{1,2} \\
u_{2}(\tau)= & u_{2,2} .
\end{array} \quad \tau \in[\bar{\delta}, 2 \bar{\delta})
$$

Integration of the state equations gives us $z(2 \bar{\delta})$ as a function of $z(\bar{\delta}), u_{1,2}, u_{2,2}$. Using $z(\bar{\delta})$ from above, we get an expression for $z(2 \bar{\delta})$ in terms of $z(0), u_{1,1}, u_{1,2}, u_{2,1}, u_{2,2}$. This procedure of piecewise integration and substitution can be repeated as many times as necessary.

For path planning, we choose to keep $u_{1}$ at a constant value over the entire trajectory. We therefore iterate the equations for $z(\bar{\delta})$ $m-1$ times so as to have exactly $m$ parameters for which to solve: $u_{1}, u_{2,1}, \ldots, u_{2, m-1}$. The total time needed for steering is $\delta=(m-1) \bar{\delta}$. Although $\delta$ can be chosen arbitrarily, a smaller time $\delta$ will result in larger inputs $u$ to achieve the same path.

The $m$ equations which result from setting $z(0)=z^{i}$ and $z(\delta)=z^{f}$ are polynomial (of order $m-2$ ) in $u_{1}$ but are linear in $u_{2,1}, \ldots, u_{2, m-1}$. Since $u_{1}$ is easily determined from

$$
u_{1}=\left(z_{1}^{f}-z_{1}^{0}\right) / \delta
$$

the remaining $m-1$ linear equations can be solved for $u_{2}$ quite easily. This is one of the reasons that we propose keeping $u_{1}$ constant over the entire trajectory; if $u_{1}$ varied, we would need to solve high-order polynomial equations in the $u_{1, k}$ parameters.

It should be noted that if $z_{1}^{j}=z_{1}^{i}$, or the initial and final states agree in the first coordinate, this method as stated so far will fail to yield a solution. From looking at the chained form equations, it is obvious that if $u_{1}=0$, only the second state $z_{2}$ can move; all other states must remain stationary. In practice, this case is dealt with by planning two paths, the first of which takes the initial condition to an 
intermediate state, the second of which joins the intermediate state with the goal position. The concatenation of these two paths is a valid trajectory between the start and goal. Our algorithm chooses the intermediate point $z^{m}$ halfway between the initial and final points in all coordinates except the first, which we choose to be offset from the starting position by a constant amount, where the constant offset can be adjusted to fit the situation.

Another reason for choosing $u_{1}$ to be constant over the entire trajectory is that in the mobile robot and trailer system, this input is roughly equivalent to the driving velocity. Because of the coordinate transformation that maps $u_{1}$ to the actual velocity $v_{0}$, the actual velocity of the robot will not be constant, but in most cases it will not cross zero and change sign. This means that the robot will not have to execute backing-up maneuvers to achieve its final goal position.

The main drawback of the piecewise constant inputs is the discontinuity of $u_{2}$. The models used in this paper are purely kinematic using as inputs the driving and steering velocities. In a real robot system, the inputs are not velocities but accelerations, or torques. When a path satisfying the velocity constraints is found, the input velocities need to be differentiated to find the corresponding accelerations. Of their very nature, the piecewise constant trajectories are not differentiable at the switching points.

4.3 Other Choices

Yet another possibility for steering systems in chained form is to use polynomial inputs:

$$
\begin{aligned}
& u_{1}=1 \\
& u_{2}=c_{0}+c_{1} t+\cdots+c_{m-2} t^{m-2} .
\end{aligned}
$$

This approach has the advantage of a constant input on $u_{1}$ with the added advantage of the differentiability of $u_{2}$.

The time needed to steer the system from $z^{i}$ to $z^{f}$ is determined by the change desired in the first coordinate,

$$
T=z_{1}^{f}-z_{1}^{0} \text {. }
$$

Once $T$ has been found, the chained form equations can be integrated using the initial condition $z(0)=z^{i}$ and then evaluated at time $T$. Setting $z(T)=z^{f}$ yields a total of $m-1$ equations affine in the $m-1$ variables $c_{0}, \ldots, c_{m-2}$,

$$
M(T)\left[\begin{array}{c}
c_{0} \\
\vdots \\
c_{m-2}
\end{array}\right]+f(z(0), T)=\left[\begin{array}{c}
z_{2}^{f} \\
\vdots \\
z_{m}^{f}
\end{array}\right]
$$

where the matrix entries $M_{i, j}(T)$ have the form:

$$
M_{i, j}=\frac{(j-1) ! T^{i+j-1}}{(i+j-1) !} .
$$

It may be shown that this matrix is nonsingular for $T \neq 0$.

Note that if $z_{1}^{\prime}-z_{1}^{i}<0$, then we get a solution which gives a negative time period. This situation is easily remedied by choosing $u_{1}=-1$.

As in the case of steering with piecewise constant inputs, this method will yield no solution when $z_{1}^{f}-z_{1}^{i}=0$. We follow the same procedure outlined in Section 4.2 to deal with this case.

Because of the simple form of the chained form system, many different classes of input functions other than the three described above could be used to steer systems in this form. The chief requirement is that there should be at least as many parameters in the input functions as there are states. For multi-trailer systems, a desirable characteristic of the input functions is that $u_{1}$ have few or no zero-crossings since these will correspond to fewer backups. In fact, the number of backups needed to complete a manoeuver may be taken as a measure of complexity of an input class.

\section{Simulations and Observations}

We now have an extensive toolbox from which to choose for steering an $N$-trailer system. With two different coordinate transformations into chained form, and at least three different methods for steering the system once it is in chained form, we can try to pick the best combination of coordinate transformation and input type for each start and goal point. There is as yet no formal way to define when one path is "better" than another, but as we mentioned earlier, we tend to think of desirable paths as those that have few backups and do not stray too far from the vicinity of the start and goal points.
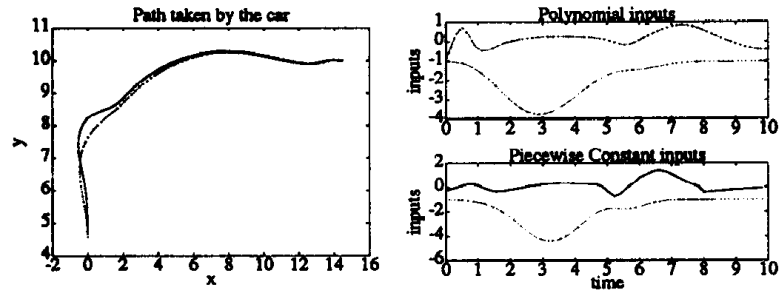

Figure 5: Backing a car with two trailers into a loading dock. Here are trajectories found by two different steering methods for the same initial and final conditions: the solid line corresponds to the piecewise constant inputs and the dashed line to the polynomial inputs. The input $v_{0}$ is the dotted line in both graphs. The $x, y$ trace of the front axle of the car is shown on the left.

One of the things that must be considered is coordinate singularities. Although we have shown that all three methods proposed here will find a path between any start and goal points in the chained form coordinates, there is no guarantee that this path, when transformed back into the actual coordinates, will avoid the transformation singularities. If a singularity does result, another steering method might yield a valid path, or perhaps an intermediate point will need to be chosen, and the path planned in two or more steps.

In Figures 5 and 6 , we show two different paths for a front-wheel drive car with two trailers. We have chosen the wheelbase of the car to be $L_{1}=0.5$ units, and each trailer to have a length of $L_{2}=L_{3}=2$ units. Each path was generated by transforming the start and goal points into the chained form coordinates, steering the chained form system using one of the methods from Section 4, and finally, transforming the trajectory back into the original coordinates.

The trajectory shown in Figure 5 represents the truck backing into a loading dock. The initial condition is $\left(x_{3}, y_{3}, \theta_{3}, \theta_{2}, \theta_{1}, \theta_{0}\right)=$ $(10,10,0,0,0,0)$ and the final position is $\left(0,0, \frac{\pi}{2}, \frac{\pi}{2}, \frac{\pi}{2}, \frac{\pi}{2}\right)$. Coordinate transformation 2 is used since the first coordinate transformation is singular at the goal position. We have presented the trajectory of the front of the car $\left(x_{0}, y_{0}\right)$ instead of the back of the second trailer $\left(x_{3}, y_{3}\right)$ to amplify the difference between the two steering methods; the trajectories of the second trailer are virtually identical.

In Figure 6 we again present the path taken by the front car, for two different coordinate transformations but the same steering method. The trajectories in the chained form coordinates are identical; however, a difference can be seen in the physical coordinates. Once again, the trajectory traced by the rear of the second trailer is very similar in both cases. Some scenes from a movie animation of this trajectory are shown in Figure 7.

With the sinusoidal steering method, there is one parameter that can be adjusted independently of the start and goal positions; this is the magnitude of the sinusoid on the first input, or $a_{1}$ in the terminology of Section 4.1. In constructing this movie, we examined several different values of $a_{1}$; a larger value of $a_{1}$ will correspond to the car driving out farther before it starts backing into the space. We were able to choose a value for this parameter so that the car and trailer system did not hit any of the obstacles along its path.
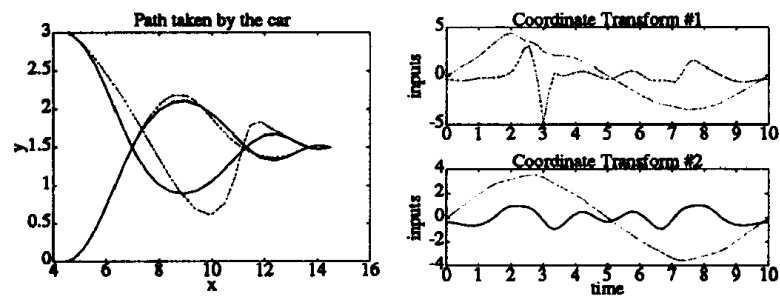

Figure 6: Parallel-parking a car with two trailers using sinusoids. The trace of the front car is shown on the left for two different choices of coordinates: Transformations 1 (solid line) and 2 (dashed line). We also see how the steering input differs on with the two transformations, although for this path, the driving input $v_{0}$ (dotted line) is similar in both cases. 


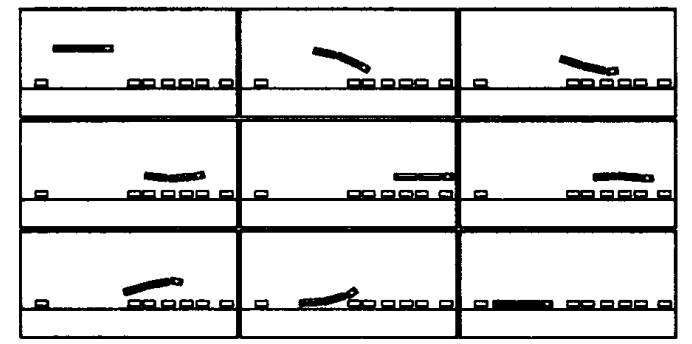

Figure 7: Scenes from a movie animation, showing the front-wheel drive car with two trailers parallel-parking in the presence of obstacles. Sinusoidal inputs were used for steering. The first coordinate transformation was used.

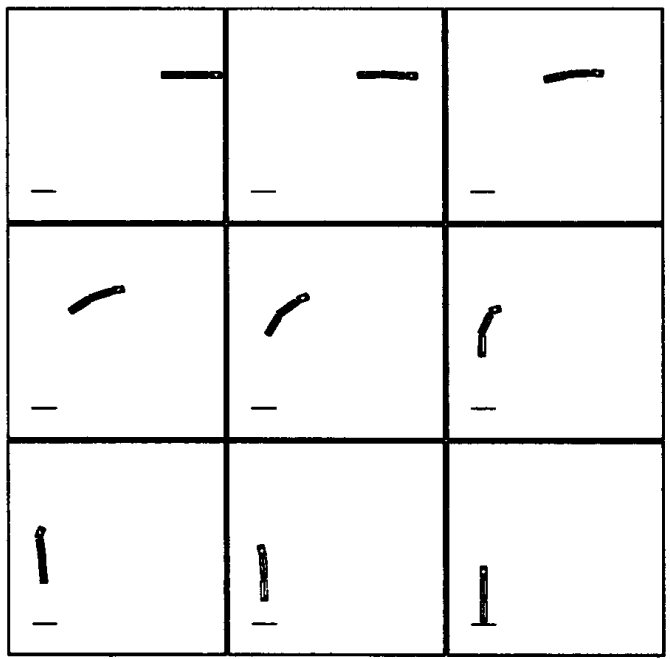

Figure 8: Scenes from a movie animation, showing the front-wheel drive car with two trailers backing into a loading dock. Piecewise constant inputs were used to steer the chained form system.

\section{Conclusion}

In this paper we applied the machinery of exterior differential systems to the $N$-trailer problem. We showed that the multi-trailer system could be put into Goursat normal form, and that this is the dual to chained form. We solved the motion planning problem for the mobile robot pulling $n$ trailers by converting the kinematic equations into chained form, and steering the chained form system from an initial to a final position, then converting the trajectory back into the original coordinates.

This work has several natural avenues of continuation, including the generation of trajectories for the $N$-trailer system in an environment cluttered with obstacles as in $[11,13]$, the stabilization of open loop trajectories, perhaps using a technique such as that outlined in [23], and the study of generalized Goursat canonical forms for exterior differential systems of higher codimension as discussed in $[3,7,15]$. In addition, there are nonholonomic systems whose constraints fail to meet the conditions of the Goursat normal form, for example, the system modeling a circular finger tip rolling on a planar face [17]. The problem of steering such systems remains an open one.

\section{Acknowledgements}

We would like to thank several people who have helped us in understanding this problem over the past few years: R. Brockett, L. Bushnell, J. Canny, R. Gardner, G. Giralt, G. Lafferiere, J-P. Laumond, Z. Li, B. Mirtich, R. Montgomery, D. Normand-Cyrot, W. Sluis, W. Shadwick, O. Sørdalen, H. Sussmann and G. Walsh.

D. Tilbury and S. Sastry would like to thank S. Mitter for his hospitality at LIDS and CICS at MIT where some of this research was done in the Fall of 1992.

\section{References}

[1] J. Barraquand and J-C. Latombe On nonholonomic mobile robots and ptimal maneuvering. In th Intl. Symp. Int. Control, pp. 74-83, 1989.

[2] R. Bryant, S. Chern, R. Gardner, H. Goldschmidt, and P. Griffiths. Exterior Differential Systems. Springer-Verlag, 1991.

[3] L. Bushnell, D. Tilbury, and S. Sastry. Extended Goursat normal forms with applications to nonholonomic motion planning. $C D C 1993$.

[4] R. Chatila. Mobile robot navigation: Space modeling and decisional processes. In O. Faugeras and G. Giralt, eds., Robotics Research: The Third Intl. Symp., pp. 373-378. MIT Press, 1986.

[5] A. Divelbiss and J. Wen. A global approach to nonholonomic motion planning. $C D C$ 1992, pp. 1597-1602.

[6] M. Fliess, J. Lévine, P. Martin, and P. Rouchon. On differentially flat nonlinear systems. Proc. NOLCOS, pp. 408-412, 1992.

[7] R. Gardner and W. Shadwick. The GS algorithm for exact linearization to Brunovsky normal form. IEEE Thans. Auto. Control, 37(2):224-230, 1992.

[8] G. Giralt, R. Chatila, and M. Vaisset. An integrated navigation and motion control system for autonomous multisensory mobile robots. In M. Brady and R. Paul, eds., Robotics Research : The First Intl. Symp., pp. 191-214. MIT Press, 1984.

[9] J-P. Laumond. Nonholonomic motion planning versus controllability via the multibody car system example. Tech. Report STAN-CS-90-1345, Stanford Dept. Computer Science, December 1990.

[10] J-P. Laumond. Controllability of a multibody mobile robot. In Proc. of the Intl. Conf. on Advanced Robotics, pp. 1033-1038, 1991.

[11] J-P. Laumond, P. Jacobs, M. Taï, and R. Murray. A motion planner for nonholonomic mobile robots. IEEE Trans. Robotics and Automation, 1993. In press.

[12] Z. Li and J. Canny, eds., Nonholonomic Motion Planning. Kluwer, 1993.

113] B. Mirtich and J. Canny. Using skeletons for nonholonomic path planning among obstacles. In Proc. IEEE Intl. Conf. Robotics and Automation, among obstacles. In

[14] S. Monaco and D. Normand-Cyrot. An introduction to motion planning under multirate digital control. CDC 1992, pp. 1780-1785.

[15] R. Murray. Nilpotent bases for a class of non-integrable distributions with applications to trajectory generation for nonholonomic systems. Tech. Report CIT/CDS 92-002, Caltech, 1992

[16] R. Murray and S. Sastry. Grasping and manipulation using multifingered robot hands. In R. W. Brockett, ed., Robotics: Proc. Symposia in Appl. Math., Vol. 41, pp. 91-128. AMS, 1990.

[17] R. Murray and S. Sastry. Nonholonomic motion planning: Steering using sinusoids. IEEE Trans. Auto. Control, 38(5):700-716, 1993.

[18] J. Reeds and L. Shepp. Optimal paths for a car that goes both forwards and backwards. Pacific J. Math., 145(2):367-393, 1990.

[19] C. Samson. Velocity and torque feedback control of a nonholonomic cart. In Intl. Workshop in Adaptive and Nonlinear Control: Issues in Robotics, pp. 125-151, 1990.

[20] O. Sørdalen. Conversion of the kinematics of a car with $\mathrm{N}$ trailers into a chained form. In Proc. IEEE Intl. Conf. Robotics and Automation, pp. 382-387, 1993.

[21] D. Tilbury, J-P. Laumond, R. Murray, S. Sastry, and G. Walsh. Steering car-like systems with trailers using sinusoids. In Proc. IEEE Intl. Conf. Robotics and Automation, pp. 1993-1998, 1992.

[22] D. Tilbury, R. Murray, and S. Sastry. Trajectory generation for the Ntrailer problem using Goursat normal form. Tech. Report UCB/ERL M93/12, ERL, UC-Berkeley, 1993.

[23] G. Walsh, D. Tilbury, S. Sastry, R. Murray, and J-P. Laumond. Stabilization of trajectories for systems with nonholonomic constraints. In Proc. IEEE Intl. Conf. Robotics and Automation, pp. 1999-2004, 1992. To appear in IEEE Trens. Auto. Control, Nov. 1993. 